Series editor: David Taylor

\section{Antique ophthalmic instruments and books: the Royal College Museum}

\section{R Keeler}

\section{Part I Instruments}

t is perhaps appropriate that an antique ophthalmic instrument and book collection should be assembled at the Royal College of Ophthalmologists in London during the 150th anniversary of Hermann von Helmholtz's discovery of the greatest of ophthalmological inventions, the ophthalmoscope.

Until 1851, ophthalmologists had struggled to explain certain eye conditions where there was a dimness or loss of vision. The use of the ophthalmoscope changed all that and a new era began with men such as Albert von Graefe, Edward Jaeger, and William Bowman at the forefront in explaining diseases that hitherto had been obscured by the impenetrable black hole of the pupil.

An early model of Helmholtz's ophthalmoscope (1851) (Fig l) takes pride of place in the Oxford Room. Beside it is the monograph Helmholtz published explaining, in some detail, the construction and use of his instrument. These are rare and valuable items and are among more than 70 ophthalmoscopes of the 19th century. This collection is probably one of the most complete to be seen anywhere.

There are a number of ways in which one can view these exquisitely made instruments. A quick survey brings home the extraordinary number of variations of this simple optical instrument. A more detailed study reveals the slow evolution of the ophthalmoscope but the most rewarding of all is to scrutinise each model in turn.

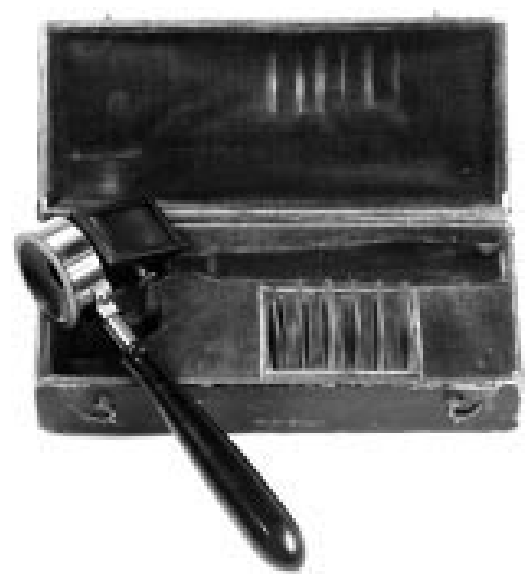

Figure 1 Helmholtz ophthalmoscope 1851.
It seems that there was no ophthalmologist worth his salt who did not have an ophthalmoscope named after him. Edward Loring states in his Textbook of Ophthalmology "Almost every ophthalmologist has taken a hand in perfecting or at least altering the instrument and from the first I have, perhaps, done more than my share." Indeed there are several of Loring's models in the collection.

To be able to use these ophthalmoscopes requires a source of illumination, a means of reflecting light into the eye, and a lens arrangement to correct an out of focus image of the patient's fundus.

Helmholtz used a flickering candle as his source of illumination. Those attempting to use this primitive source of light today will be filled with admiration for the early pioneers of ophthalmoscopy. The miniature oil lamp with condensing lens, such as the PriestleySmith, at least stabilised the source. In 1869, Lionel Beale harnessed the light source by building an oil lamp onto the observation tube of his monocular indirect ophthalmoscope (fig 2). Henry Juler, in 1885, was one of the first to use the latest technology, the incandescent bulb. He attached a miniature bulb to the head of the ophthalmoscope pointing it towards the angled mirror.

"The modes of reflecting light into the eye are manifold." Haynes Walton, in his Practical Treatise on Disease in the Eye, mentions the extraordinary variety of mirrors used by early pioneers of the ophthalmoscope, from coated plano glass to polished concave steel.

The first mirror to appear after Helmholtz's parallel superimposed glass plates was Adolf Coccius's large square plano mirror in 1853. This was shortly followed

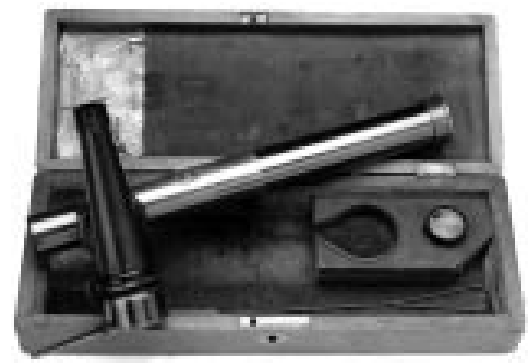

Figure 2 Beale ophthalmoscope 1869. by his concave mirror. Mirrors were made from plated glass but others were polished steel, examples being the Desmarres and Liebreich models.

The focal length of these concave mirrors also varied; those of a lower power being used for the indirect method of ophthalmoscopy introduced by Theodore Ruete in 1853. Many of the instruments shown incorporate mirrors of more than one focal length. Edward Jaeger's ophthalmoscope of 1854 has two different mirrors and a version of Helmholtz's glass plates.

John Couper of Moorfields was the first to angle the mirror, which until then lay flat against the lens track. Couper's invention allowed the observer to view the patient's fundus looking perpendicularly, without distortion, through the lens while angling the mirror in the direction of the source of illumination. There are two samples of Couper's ophthalmoscopes in the collection and it is anticipated that a rare example of his first ophthalmoscope will be loaned from the Royal College of Surgeons' collection.

There are numerous examples of Loring's rectangular mirror. He cut off the vertical sides of a round mirror allowing the operator to tilt the mirror left or right. Loring's idea was used on a number of other ophthalmoscope designs.

Until 1882 mirrors of different focal length had to be interchanged on the instrument but George Lindsay Johnson mounted his two mirrors permanently on the ophthalmoscope, rotating them around a central pivot. Andrew Stanford Morton in 1885 used the same idea in his most popular of ophthalmoscopes,

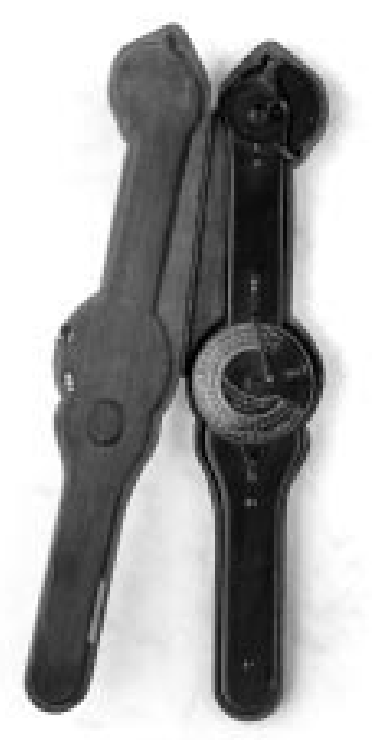

Figure 3 Couper's "chain of lenses" ophthalmoscope 1883. 
which went through many changes in its 70 year life.

If there were many ways of reflecting light into the eye it was nothing compared to the inventiveness and ingenuity employed by engineers in the use of lenses to correct for ammetropia and obtain a sharp image of the fundus.

Shortly after Helmholtz introduced his ophthalmoscope his engineer, Egbert Rekoss, invented the rotatable disc of concave and convex lenses (1852). This disc has been used ever since. But early ophthalmoscopes such as the Liebreich, Coccius, Jaeger, and Loring all had detached single lenses of varying concave and convex powers.

Early practitioners of the ophthalmoscope such as Couper and de Wecker also used it for refraction. This required the ophthalmoscope to have a wide range of lenses in small increments of power change. Couper's "chain of lenses" ophthalmoscope is a brilliant piece of engineering for it incorporates 73 miniature lenses driven around the handle and head of the instrument in a grooved track (Fig 3).

Xavier Galezowski, whose first of four different models, was a tubular monocular indirect ophthalmoscope, produced an ophthalmoscope with a single Rekoss disc but with two concentric circles of lenses in it, one with concave and the other with convex lenses (fig 4). All the

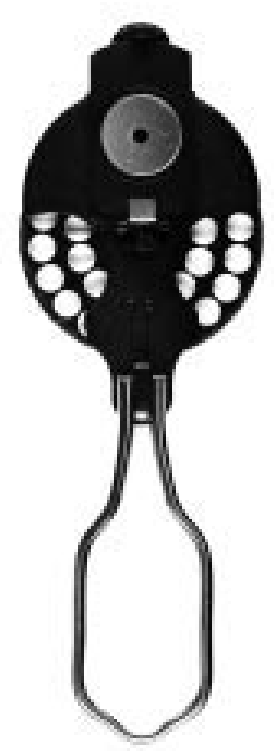

Figure 4 Galezowski ophthalmoscope 1882. operator had to do was move the whole disc up or down in a vertical direction to introduce the plus or minus lens range he wanted.

The collection includes a Roth ophthalmoscope (1894). This was the first to automate the introduction of a wide range of lenses. Although the museum does not have a sample of the very first binocular indirect ophthalmoscope designed by Giraud-Teulon (1861), it does have a Lawrence and Heisch ophthalmoscope ( 1862 ) (Fig 5). In some ways this is a more desirable instrument as one can only but admire the beautiful craftsmanship in its complex mechanism for changing the interpupillary distance compared to the solid and immovable rhomboid prism arrangement used on the Giraud-Teulon instrument.

Ophthalmoscopes at present dominate the display at the college but there are many other instruments of great interest.

There is an 1875 trial lens set by Paetz and Flohr. This was rescued a few years ago from a skip after a hospital in the eastern part of Germany cleared out its attic. With the trial case there is a handwritten sheet of paper with conversion tables for the power of the lenses. Robert Brudenell Carter, in his book A Practical Treatise on Diseases of the Eye, describes the difficulties arising at the time of the change from specifying the power of lenses by their focal length in inches to the metric system. He writes "moved by these considerations, the International Congress of Ophthalmology decided, in 1867, to appoint a committee to report upon the question; and in $1872 \ldots$ the Congress resolved to adopt a metric scale; but there were many obstacles to be overcome before this decision could be carried into effect. In the first place, the manufacturers of lenses rebelled against the proposal as a matter of commerce, and objected so strongly to having their inch grinding tools rendered obsolete, that at one time metrical lenses could not be procured. I obtained a set

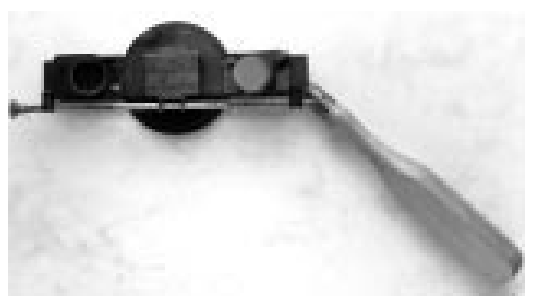

Figure 5 Lawrence and Heisch ophthalmoscope 1862. myself, nine years ago, from Paetz and Flohr of Berlin, but both they and the Paris makers, so lately as 1874 , refused to supply another set or even to make good some breakages in that which I possessed ...."

Surgical instruments of the 19th century are well represented in the collection. There are many ivory handled cataract knives, keratomes, and needles, some contained in beautifully designed wooden boxes with brass fittings. There are corneal and scleral trephines of every type as well as retractors and lacrimal probes. There is even a probe designed by Lord Nuffield for his own use.

In a separate cabinet in the Council Room there is a more varied collection of instruments which include trial frames, miniature eye magnets, folding nonluminous retinoscopes, and a variety of tonometers. Of special historical value are those items marked with the name of the owner and in a number of instances the inventor himself. For instance, there is Basil Lang's retinoscope and both the father and son, Henry and Frank Juler's own ophthalmoscopes.

On view is Claude Worth's series of five ivory balls of different sizes to assess the vision of very young children by rolling the balls, starting with the smallest, in front of the child and watching for signs of eye movement.

There are samples of the first soft contact lenses invented by Otto Wichterle (1962) which are suspended in water filled phials. Every kind of surgical loupe is represented and although not an ophthalmological instrument, there is a camera lucida of which so much has been written and commented on recently, and especially highlighted in David Hockney's book, Secret Knowledge.

In the early days of ophthalmoscopy, model eyes were used for teaching diseases of the eye and, later, refraction. Some of these have beautifully painted fundi showing various pathological conditions. The collection includes a Perrin, Landolt, Davidson, and Rylander.

Those wishing to study the lives of many of these ophthalmological instrument inventors have an opportunity to do so in the library, which will be the subject of the second part of this series.

Author's affiliations

R Keeler, curator Royal College of

Ophthalmologists, London, UK;

rkeeler@freenetname.co.uk 